\title{
Making Roads Safer: Optimizing De-icing Using Snowmelt Rates and Slope Data
}

\author{
Fletcher Chapin ${ }^{1}$, Olufemi Omitaomu², Budhendra L. Bhaduri \\ ${ }^{1}$ Cornell University \\ Ithaca, NY, USA \\ ${ }^{2}$ Oak Ridge National Laboratory \\ 1 Bethel Valley Rd, Oak Ridge, TN, USA \\ ftc22@cornell.edu; omitaomuoa@ornl.gov
}

\begin{abstract}
More than \$1.5 billion is spent annually on winter road maintenance programs in the United States. With an increase in severe winter storms, many municipalities are encountering difficulty in clearing their roads of ice and snow within their budgetary limits. Currently, roads are classified based on traffic counts, with busier (arterial) roads being treated before less busy (secondary) roads. This approach is problematic because most people live on secondary roads and must travel over these untreated roads before they can reach treated roads. In addition, inefficient distribution of de-icing material can have knock-on environmental impacts with increased material run-off into surrounding areas. To address these concerns, we proposed a road vulnerability index based on rate of snowmelt and road slope. We calculated the snowmelt rates in 1-m by 1-m squares of all roads in Knox County, Tennessee, using an empirical equation developed by the U.S. Army Corps of Engineers. This equation takes in freely available weather data and incident solar radiation, which we calculated based on LiDAR (Light Detection And Ranging) data. Using the model results, we propose a more efficient winter road maintenance strategy: apply de-icers to the steepest roads with the slowest snowmelt, rather than to the busiest roads. Under this approach, we argue that given existing budgetary constraints, more vulnerable roads can be treated with de-icing material and provide for easier mobility for motorists traveling on secondary roads. Our approach provides a more cost-effective, environmentally-conscious, and mobility enhancing application strategy.
\end{abstract}

Keywords: Winter road maintenance, snow removal, ice removal, de-icing, road salt

\section{Introduction}

With the increase in winter storm severity and activity due to climate change, municipalities have been struggling to clear roads of snow and ice within their budgetary limits. With larger, more frequent winter storms, municipalities are experiencing shortages in road salt and other de-icing material. In 2014, the Pennsylvania Department of Transportation consumed 686,000 tons of salt, 200,000 more tons than used in the average year [1]. In other areas not accustomed to heavy snow, there are inadequate snow removal vehicles to cover all roads needing treatment. To ensure mobility during winter storms, roads that experience higher traffic are treated first, with less travelled, secondary roads being treated later. The problem with this approach is the fact that motorists must travel on the untreated, secondary roads to get to the treated, major roadways. On top of the traffic issues, over-treatment of high traffic roadway can cause knock-on environmental impacts due to run-off of de-icing materials into local environments, with potential impact on wildlife and fauna [2].

Given the increase in winter storm severity due to climate change and need to conserve and utilize scarce resources, it is necessary to develop a new approach to snow and ice removal [3]. Previous research has shown that snow depth correlates with number of traffic accidents [4]. In an effort to reduce amount of money and resources poured into ensuring roads are cleared of snow and ice, we propose a road vulnerability index (RVI) that classifies road segments into three categories, Most Vulnerable, More Vulnerable, or Vulnerable, based on the snowmelt rate and slope. We calculated the snowmelt rates at 1 square meter spatial resolution for all roads in Knox County, Tennessee, using an empirical equation developed by the U.S. Army Corps of Engineers. This equation takes in freely available weather data and incident solar radiation, which we calculated based on LiDAR (Light Detection And Ranging) data. Using this index, a GPS unit could be connected to the de-icer dispensing mechanisms on trucks in order to automate the placement of de-icer substance onto road segments classified as Most Vulnerable first, with subsequent treatment of more vulnerable and vulnerable roads 
depending on availability of de-icing material. This will allow municipalities to cover more area with less money, helping both their budget and their citizens' safety.

\subsection{Model Considerations}

A number of factors were considered in the adaptation of the model that informs RVI. First, we considered the accuracy of the model. The model is based on the energy balance approach from the U.S. Army Corps of Engineers, which is considered more detailed and widely used than the alternative, the degree-day method. The reason the degree-day method persists despite its lack of detail is its simple parameters.

Second, we considered data availability to ensure we had the required values for the more detailed energy balance approach. Most of the data required was weather data, which is freely available through online sources. Solar radiation data was obtained in a preliminary study for calculating solar potential for building tops [5]. The authors in the aforementioned study used LiDAR (Light Detection And Ranging) data and the hemispherical viewshed algorithm developed by Oak Ridge National Laboratory's (ORNL) Visual-SOLAR Project for estimating rooftop solar panel potential [5].

Third, we considered what parameters outside of snowmelt to include in our model. In our development of a parsimonious model, we tried to exclude extraneous parameters, but felt further investigation was needed to understand what made a road dangerous in addition to snow accumulation. In the course of parameter selection, we chose to include additional parameter, slope, in order to maintain the model's simplicity. Additionally, elevation data is freely available and can be easily converted to slope data in GIS programs such as ArcMap.

Lastly, we selected a test area and time span. The original study area was the whole of Knox County, Tennessee. However, we ended up only classifying a small portion of roads just to the East of downtown Knoxville (see Figure 1). We calculated the snowmelt rate for the entire county, but the computational time was too large for classifying the entire county so we broke it into smaller pieces. The piece selected was chosen because it contained a mix of both busy highways and low-traffic roads. With respect to the time period of interest, we chose to model between the time frame of January $20^{\text {th }}$ and February $27^{\text {th }}$ of 2016 . This time period was chosen because the recorded snowfall in Knoxville in winter 2016 occurred between those dates.

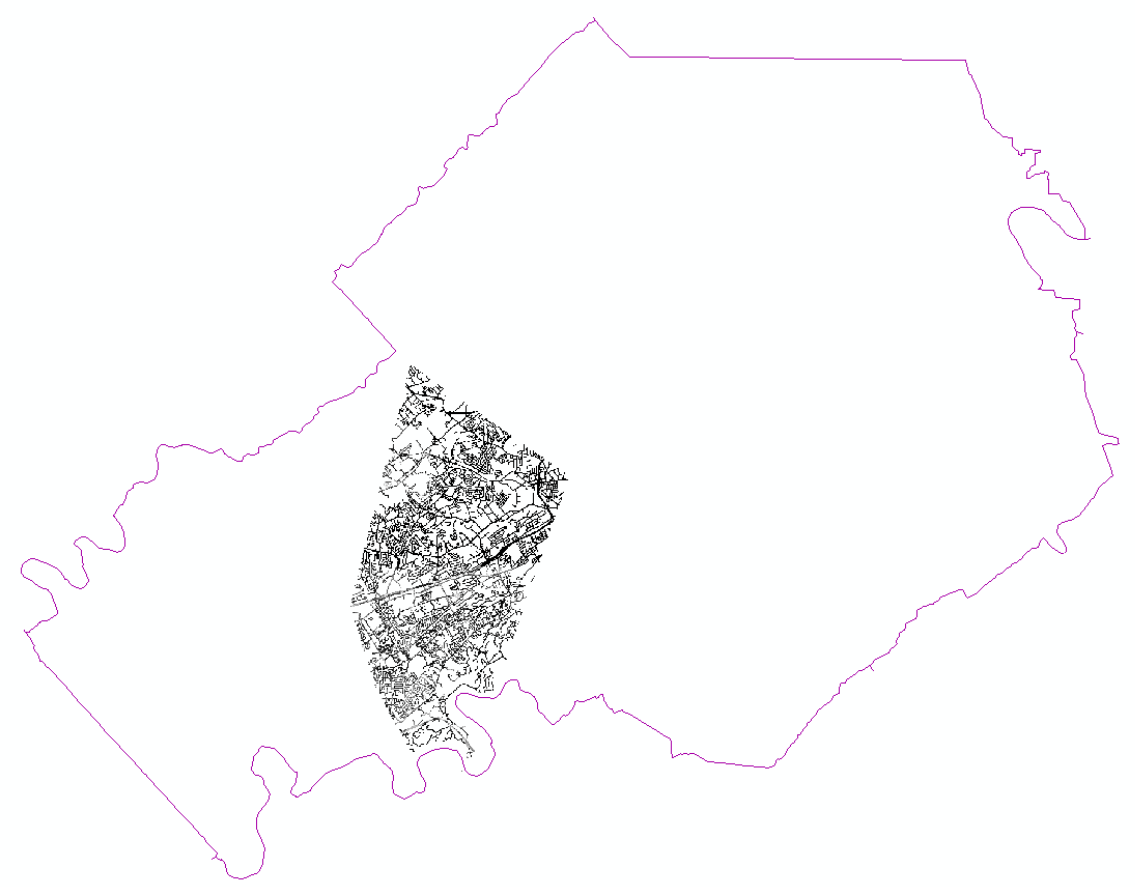

Fig. 1: Model classification area within Knox County. 


\section{Methods}

The model is used to classify all roads in the target area in 1-m by 1-m squares as Most Vulnerable, More Vulnerable, or Vulnerable. This determination is made by using our proposed classification in Table 1, whose inputs are a previous classification of Low, Medium, and High for both slope and snowmelt. We purposely classified a majority of the combinations as most vulnerable in order to skew the results towards a cautious approach which is better when dealing with life or death situations such as car accidents.

Table 1: Classification rules.

\begin{tabular}{|c|c|c|c|c|}
\hline \multirow{5}{*}{ 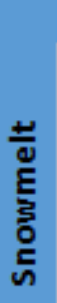 } & \multirow{5}{*}{$\begin{array}{c}\text { low } \\
\text { medium } \\
\text { high }\end{array}$} & \multicolumn{3}{|c|}{ Slope } \\
\hline & & high & medium & low \\
\hline & & most vulnerable & most vulnerable & most vulnerable \\
\hline & & most vulnerable & most vulnerable & more vulnerable \\
\hline & & most vulnerable & more vulnerable & vulnerable \\
\hline
\end{tabular}

\subsection{Snowmelt Rate Calculation}

The following equation [6], developed by the U.S. Army Corps of Engineers was used to calculate the snowmelt rate for all of Knox County during non-rainy periods:

$$
M=\mathrm{C}\left[0.002 I_{i}(1-a)+(0.0011 v+0.0145)\left(T_{a}-T_{F}\right)+0.0039 v\left(T_{d}-T_{F}\right)\right]
$$

And for rate of snowmelt during rainy periods:

$$
M=\mathrm{C}\left[0.09+(0.029+0.00504 v+0.007 P)\left(T_{a}-T_{F}\right)\right]
$$

Where $M=$ rate of melt (inches/day); $I_{i}=$ incident solar radiation on a horizontal surface (Langley/day); $a=$ albedo of snow $=\left(0.75 / D^{0.2}\right), D=$ days since last snowfall; $v=$ wind speed (miles/hour); $T_{a}=$ air temperature $\left({ }^{\circ} \mathrm{F}\right) ; T_{F}=$ freezing temperature $\left({ }^{\circ} \mathrm{F}\right) ; T_{d}=$ dew point temperature $\left({ }^{\circ} \mathrm{F}\right) ; P=$ rainfall (inches/day); and $\mathrm{C}=$ coefficient to account for variations [6].

Since the equation was empirically derived by the U.S. Army Corps, it only works if the units it was designed for are used (empirical units), so our calculations were done in empirical units and then the results were converted to SI units.

The snowmelt rate was calculated at 1-m granularity for all of Knox County using Python code, which is based on Equations 1 and 2, and data input into the equations was obtained as detailed below.

\subsection{Data Collection}

Solar data ( $I_{i}$ in equation 1) was obtained based on method in [5]. All of the weather data was downloaded as daily data from 10 personal weather stations (PWS) throughout Knox County. These PWS are spread throughout the county in order to determine whether certain sections of the county have different enough weather to substantially affect snowmelt rate. For the calculations the Voronoi Method was applied to map each 1-m square section of road to the closest PWS, so that the input to the model used the closest weather data for each section (see Figure 2).

In order for the equation to compute in Python, some SI units were converted to empirical units prior to computation. For example, input solar data in $\mathrm{kWh} / \mathrm{m}^{2} / \mathrm{day}$, which was subsequently converted to Langley/day. Additionally, we converted the output from in/day of snowmelt to $\mathrm{cm} / \mathrm{hr}$. Finally, we used $C=1$ for every calculation because the constant accounts for variation, but road surfaces are relatively constant throughout the county.

Slope data, which was not used in the model but for the classification system, was obtained by downloading elevation data from the USGS National Map database and using the Slope tool in ArcMap to convert the DEM to a raster of slope (in degrees). 


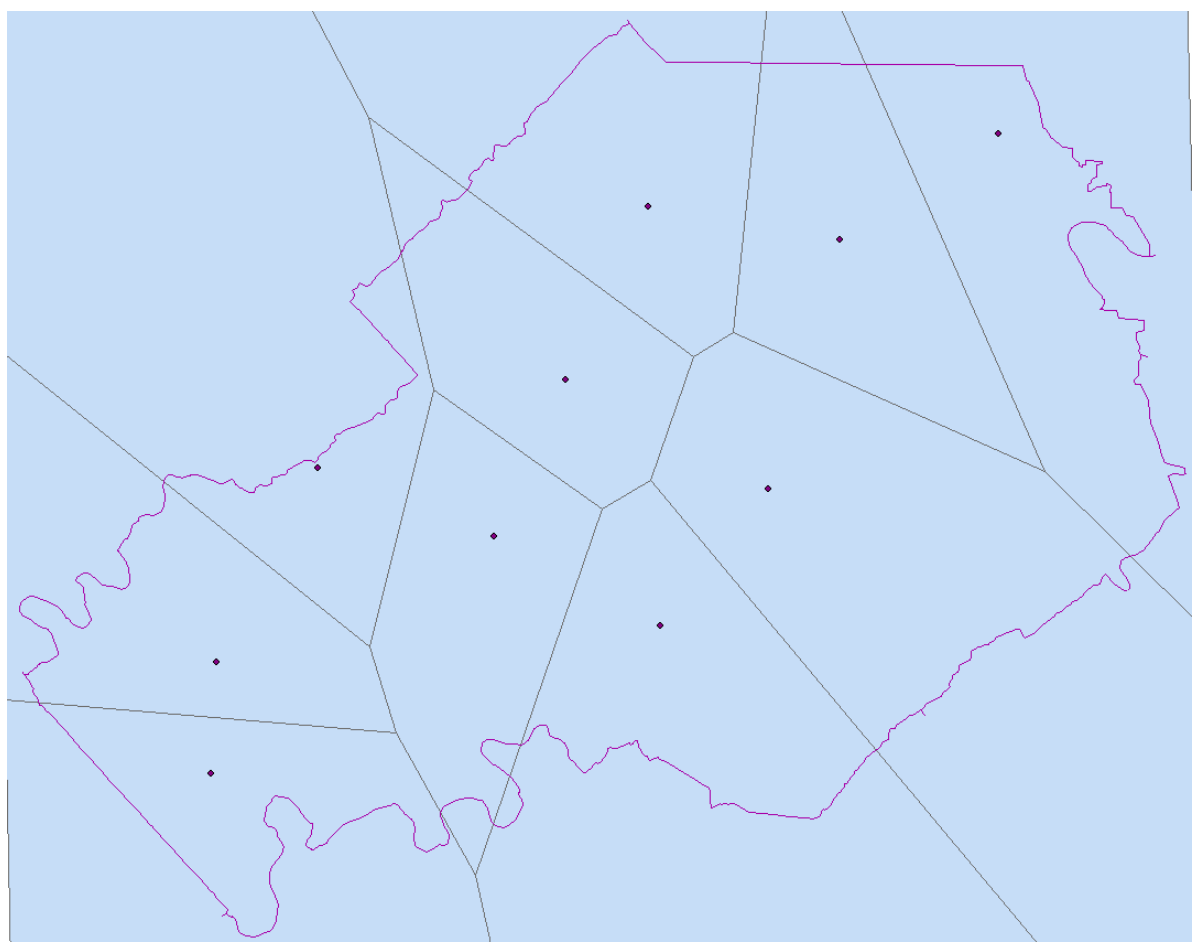

Fig. 2: Results of applying Voronoi Method to PWS.

\subsection{Classification}

Even though snowmelt rate was calculated for all of Knox County only a small section was classified. The classification step was done in R software environment. First, every road section was classified in both slope and snowmelt rate as Low, Medium, or High based on heuristic. After classifying each road section as Low, Medium, or High in each category, we then used the secondary classification system in Table 1 to determine a safety rating.

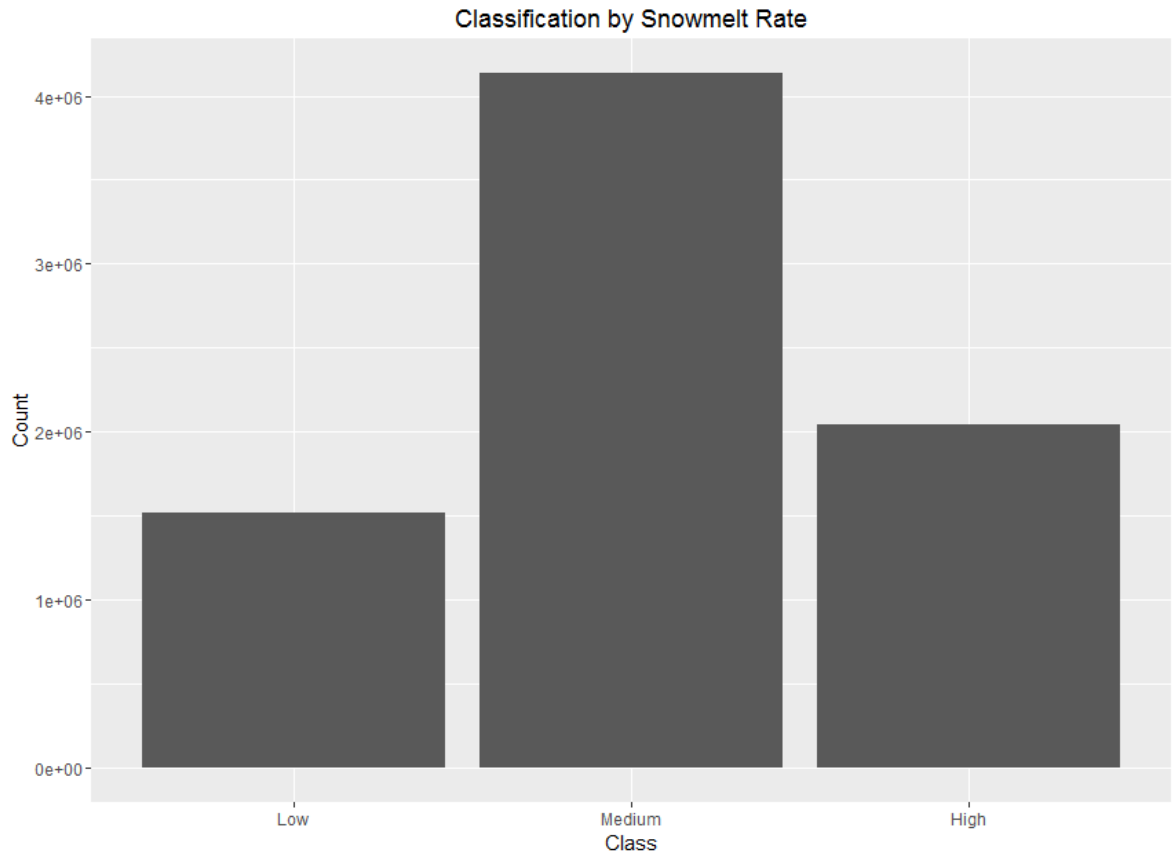

Fig. 3: Preliminary snowmelt classification. 


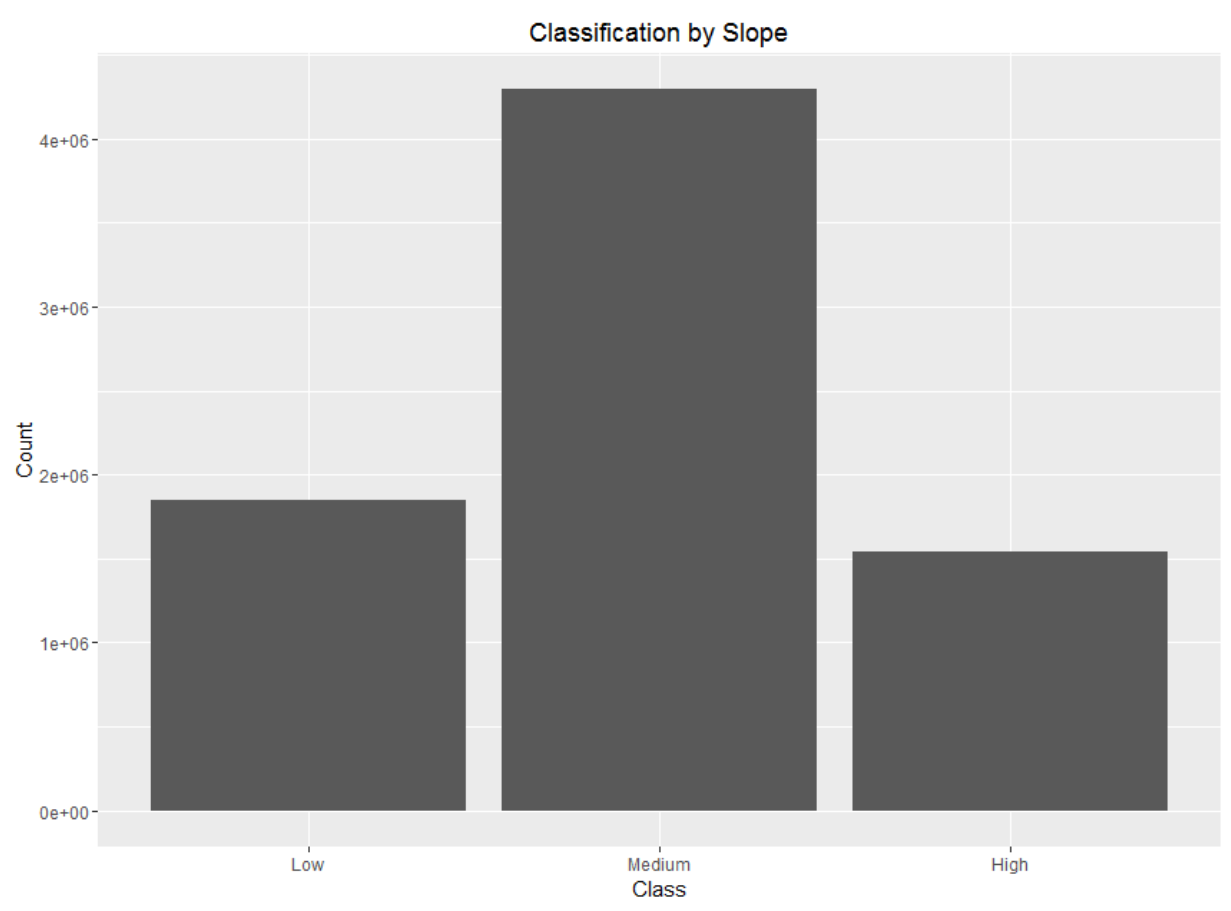

Fig. 4: Preliminary slope classification.

\section{Results}

\subsection{Snowmelt Calculation Results}

Before classifying the sample section of Knox County, we looked at the results of just the snowmelt rate calculation. The average snowmelt rate was rarely zero. The snowmelt rate then peaks around $0.55 \mathrm{~cm} / \mathrm{hr}$. and exhibits a right-hand long tail, which can be explained by some anomalous, high-melt-rate locations. Similar classification based on slope values is shown in Figure 4 . The number of $1 \mathrm{~m}$ by $1 \mathrm{~m}$ cells for rate of snowmelt between 0 and $4 \mathrm{~cm} / \mathrm{hr}$. is shown in Figure 5 .

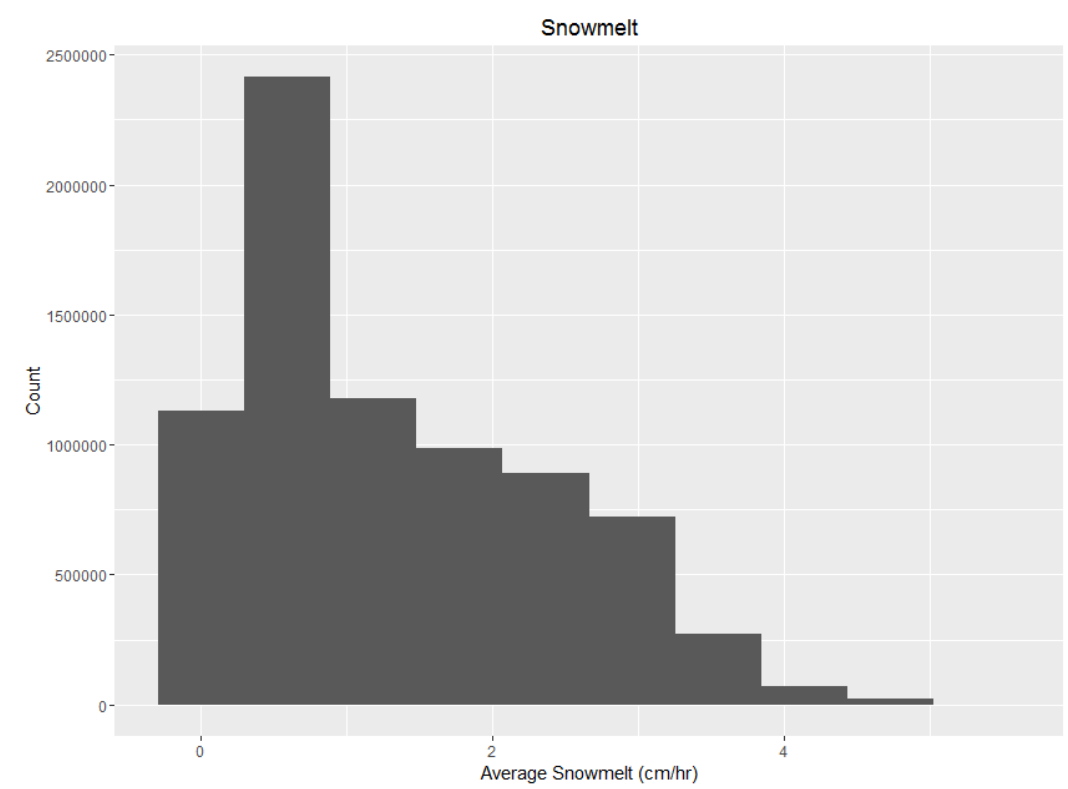

Fig. 5: Average snowmelt rate. 
The most important part of the modelling is determining which locations that are currently treated do not need to be, and which untreated locations have the most need for treatment. To this end, we developed a raster map of snowmelt rates that is split at the same cut-offs as the Low, Medium, and High classification system we used.

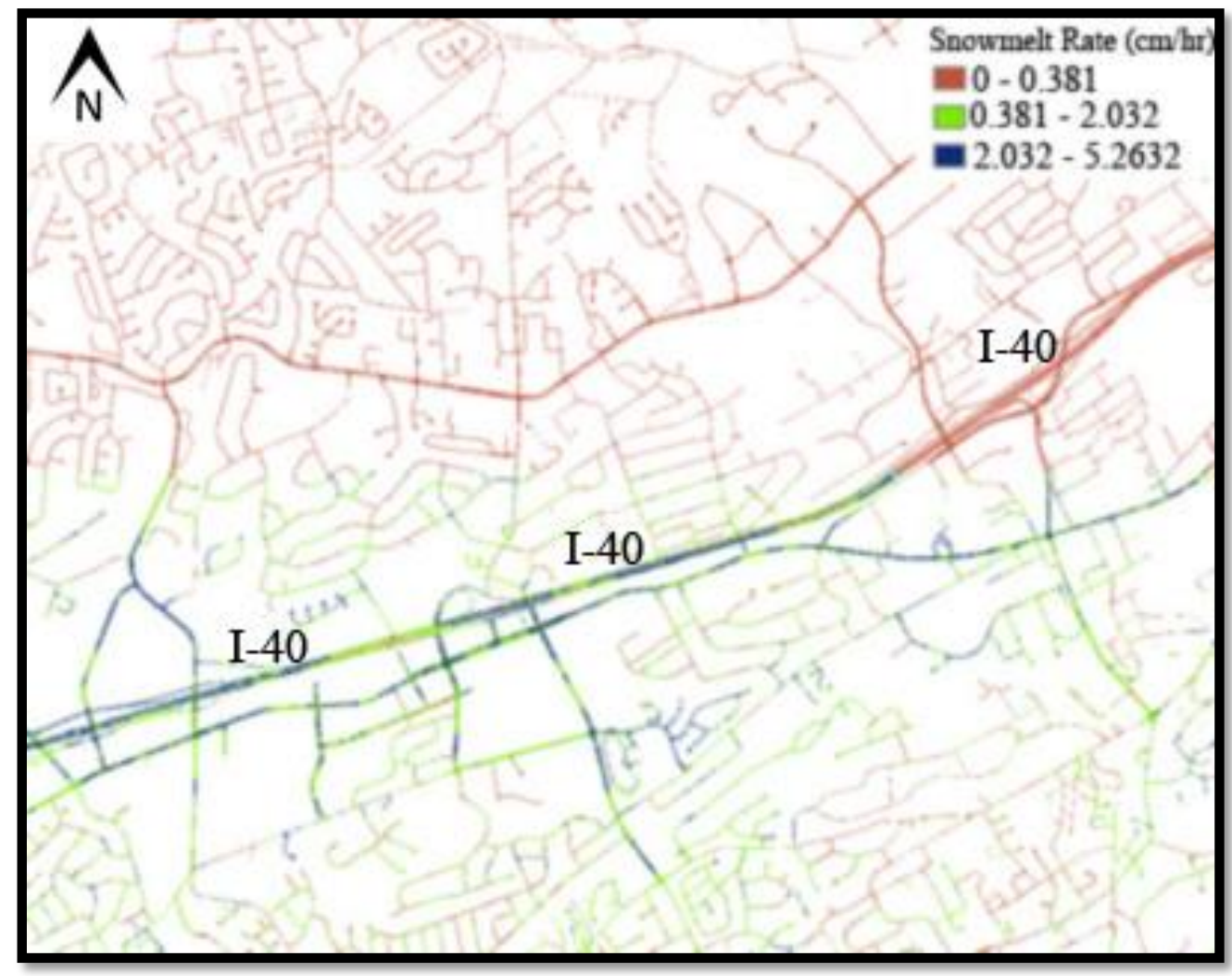

Fig. 6: Subset of raster data.

The sample of raster data (Figure 6) shows Interstate 40 (I-40) running through the centre with secondary roads on either side. We observed that the roads south of the interstate have higher snowmelt rates than those to the north. They get less solar radiation, most likely because on the south of I-40 is a commercialized area with many parking lots and stores, whereas to the north is suburban neighbourhoods which have more trees and other shading agents.

Most interestingly, portions of I-40 have the highest snowmelt rates. They are also the busiest road segments, so under the current system they receive the most salt. Under our model, as long as they are relatively flat, the salt used on these sections of road could be apportioned to the lower-melt-rate suburban roads to the north given their vulnerability under our typology.

\subsection{Classification Results}

Although we only had time to create a raster of snowmelt, not safety classifications, the results are still useful. Figure 7 shows that a majority of roads are classified as Most Vulnerable and would need to receive somewhat more salt. However, even if we assume only a small portion of the raster in Figure 6 represents flat areas, there is still a large amount of salt that could be taken from the More Vulnerable and Vulnerable sections and placed on the Most Vulnerable sections, and possibly move them into More Vulnerable or Vulnerable snowmelt rates. When millions of dollars are spent towards salt, even a small adjustment could have a substantial impact on the budget. 


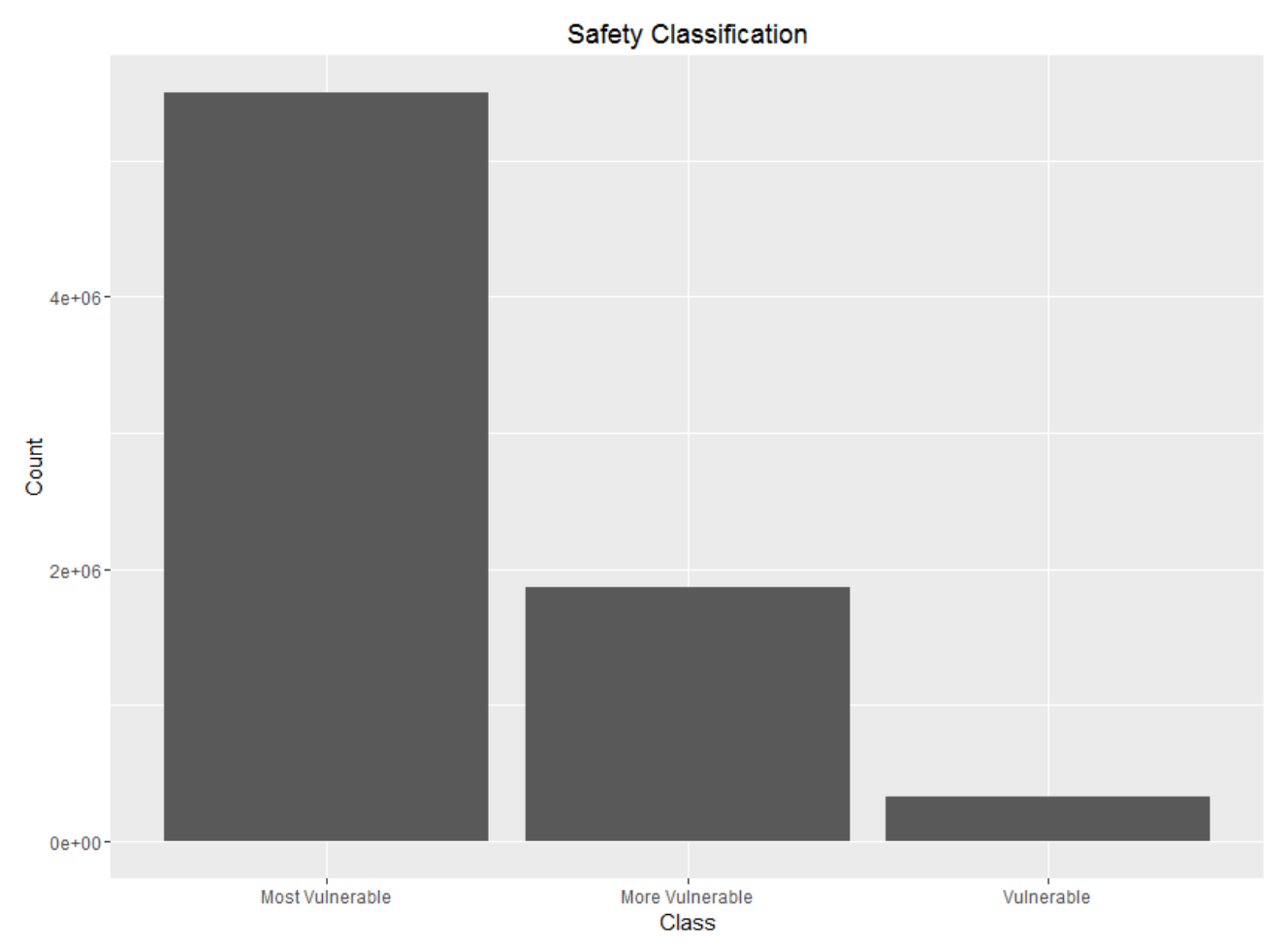

Fig. 7: Safety classification.

\section{Conclusion and Future Work}

The goal of this work was to optimize de-icer distribution on roads in order to save money, increase safety, and protect the environment. Although more future work is needed to improve the model, the preliminary results show that it is effective in calculating snowmelt rate throughout the target area and then combining that snowmelt rate with slope in order to classify the road's safety.

The most interesting results came from the examination of snowmelt rate as a raster map. The map clearly showed that portions of Interstate 40, which as the busiest road under the current de-icing guidelines would receive salt first, have the highest rate of snowmelt. This is a promising result that conveys how much improvement can be made in the methods for de-icer application. Using a classification system like the one proposed, possibly with some additional parameters, in designing snow and ice removal systems will be vital in the future to cost-effectively keep drivers safe as winter storm severity continues to increase.

There are a number of next steps that could be taken in the development of this model. First, the mapping of the safety classification similar to the snowmelt raster (Figure 6) will be important in verifying the results of this model. Second, applying this model to another region, especially one that gets more yearly snow, would be useful in verifying the results. Another possibility for additional verification is doing field studies of snowmelt rates or traffic incidents. Third, adding more parameters could be useful in designing a better classification system. Although with this step it is important to ensure that the parameters added keep the model simple and do not pose any difficulty in getting data. One parameter we are particularly interested is speed limit; we believe that drivers, especially those unfamiliar with wintery conditions, will drive faster in high speed limit areas, making it more dangerous when roads are icy. Fourth, we would be interested in investigating how the rate of snowmelt is affected by adding salt or other de-icing chemicals. Previous work on determining how much salt to apply to roads could be useful in this next step [7]. After determining the amount of ice needed to clear the roads based on the effect of salt on snowmelt rate, the road area that can be treated within certain budgets can be calculated. This could be a useful tool for governments budgeting for de-icing chemicals. Finally, the end product of this model would be to integrate it into a GPS unit so that salt trucks automatically apply de-icer on the locations deemed most vulnerable and don't apply salt on safe locations. This step is key to the practical applications of the model outputs and achieving the positive effects that the preliminary results indicate are possible. 


\section{Acknowledgements}

This manuscript has been authored by UT-Battelle, LLC under Contract No. DE-AC05-00OR22725 with the U.S. Department of Energy. The United States Government retains and the publisher, by accepting the article for publication, acknowledges that the United States Government retains a non-exclusive, paid-up, irrevocable, world-wide license to publish or reproduce the published form of this manuscript, or allow others to do so, for United States Government purposes. The U.S. Department of Energy SULI program provided summer internship support for the first author. The authors thank David Caleb Robinson for converting our R code into Python in order to reduce the runtime.

\section{References}

[1] J. Black, D. Arkin (2014, Feb. 5). "Pinched: Salt Shortage Leads to Dangerously Slippery Streets," NBC News [Online]. Available: http://www.nbcnews.com/storyline/deep-freeze/pinched-salt-shortage-leads-dangerouslyslippery-streets-n22516

[2] NH Department of Environmental Services, "Environmental, Health and Economic Impacts of Road Salt," 2016. [Online]. Available: http://des.nh.gov/organization/divisions/water/wmb/was/salt-reduction-initiative/impacts.htm

[3] A. K. Andersson, L. Chapman, "The impact of climate change on winter road maintenance and traffic accidents in West Midlands, UK," Accident Analysis and Prevention, vol. 43, pp. 284-289, 2011.

[4] J. Seeherman, Y. Liu, "Effects of extraordinary snowfall on traffic safety," Accident Analysis and Prevention, vol. 81, pp. 194-203, 2015.

[5] J. B. Kodysh, O. A. Omitaomu, B. L. Bhaduri, B. S. Neish, "Methodology for estimating solar potential on multiple building rooftops for photovoltaic systems," Sustainable Cities and Society, vol. 8, pp. 31-41, 2013.

[6] United States Army, Corps of Engineers, HEC-1 flood hydrograph package. Davis, CA: Hydrol. Eng. Ctr., pp. 283, 1998.

[7] W. R. Trenouth, B. Gharabaghi, N. Perara, "Road salt application planning tool for winter de-icing operations," Journal of Hydrology, vol. 524, pp. 401-410, 2015. 